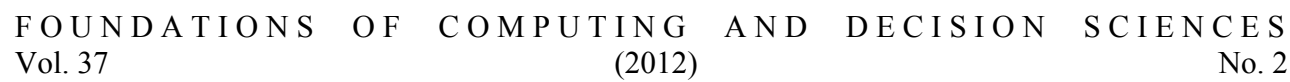

DOI: $10.2478 / \mathrm{v} 10209-011-0005-\mathrm{x}$

\title{
A PETRI NET BASED MODEL OF OXIDATIVE STRESS IN ATHEROSCLEROSIS
}

\author{
Dorota FORMANOWICZ1 ${ }^{\text {, Adam } \mathrm{KOZAK}^{2} \text {, Piotr FORMANOWICZ }}$ F $^{2,3}$
}

\begin{abstract}
In this paper a Petri net based model of the process of oxidative stress in atherosclerosis is presented and analyzed. Model expressed in the language of Petri net theory have, on one hand, an intuitive graphical representation, and on the other hand their formal properties can be analyzed using rigorous mathematical methods. Moreover, the behavior of a net can be simulated what supports the process of model development and an interpretation of the results of the analysis. Both the analysis and the simulation can be supported by many freely available software tools. In the case of biological systems an analysis the t-invariants is especially important since they correspond to some elementary biological subprocesses. In this paper the results of such an analysis are presented. In particular, minimal t-invariants, MCT-sets and t-clusters are calculated, their biological meaning is determined and some biological conclusions are drawn.
\end{abstract}

Keywords: biological systems, atherosclerosis, oxidative stress, modeling, Petri nets, t-invariants

\section{Introduction}

Computational biology is an interdisciplinary branch of science evolving on the border between biology, mathematics and computer science. Its goal is to develop mathematical models of biological processes and structures, and algorithms based on these models supporting an analysis of biological phenomena.

The impressive discoveries in the area of biological sciences during the last two decades made it clear that for deep understanding of the structure and functionality of

\footnotetext{
${ }^{1}$ Department of Clinical Biochemistry and Laboratory Medicine, Poznań University of Medical Sciences, Poznań, Poland

${ }^{2}$ Institute of Computing Science, Poznań University of Technology, Poznań, Poland

${ }^{3}$ Institute of Bioorganic Chemistry, Polish Academy of Sciences, Poznań, Poland

*Corresponding author, e-mail: piotr@cs.put.poznan.pl
} 
living organisms an application of formal mathematical and computer science methods is necessary.

Moreover, it becomes more and more clear that living objects (i.e. cells, tissues, organs, organisms etc.) are complex systems whose behavior follows not only directly from properties of their building blocks but also from interactions among these blocks. In biological systems the nets of such interactions are especially dense what is the source of complexity of them being most probably one of the fundamental properties of living organisms. It leads to a new approach to study biological phenomena based on the systems sciences point of view.

Until recently another approach, which could be called the "reductionistic", one was dominant. It focused on a precise analysis of the above mentioned basic building blocks without considering most of the interactions between them. This classical approach was in fact very fruitful and resulted in many spectacular discoveries in biological sciences, especially in molecular biology. But now it seems that this approach has serious limitations and for continuing the rapid development of biological sciences the systems approach is necessary.

Obviously, in general, this approach is not new in other disciplines, especially in technical sciences, since systems as mathematical objects are studied for years in the are of systems sciences and the results are applied in many branches of technical sciences. But in the area of biology it is something new. However, it is worth to mention that systems sciences have their roots in biology, at least to some extent. Indeed, Ludwig von Bertalanffy, the founder of general systems theory, postulated the need of studying general properties of systems inspired by his research in the area of biology in the 1920s (see [1]). Also Norbert Wiener, father of cybernetics was inspired by some biological problems when was working on the basic ideas of this new branch of science in the mid 1940s [13]. But then, the systems sciences evolved mainly in the direction of technical sciences. So, it could be said that now the systems sciences return to their biological origins what results in a discipline called systems biology (c.f. $[3,11])$.

It is worth to note that biological systems are the most complex ones studied by humans what makes its analysis extremely difficult. Moreover, contrary to technical systems, the biological ones are not constructed by humans what results in the fact that even building an exact model of such a system of moderate size is a hard and challenging task. Obviously, such a model is a necessary basis for further analysis of the system.

Usually, the models of biological processes were expressed as systems of differential equations. Models of this type are, in principle, very precise, but in the context of biological systems they have several limitations. One of them follows from the limited availability of quantitative data which are required in these models. Hence, some other methods of describing biological systems are investigated. Among them the ones based on graph theory are relatively well-suited and promising. In a natural way they describe the basic biological objects as vertices and relations between them as edges or arcs. Here, Petri nets seem to be especially interesting.

Nets of this type have been proposed in the early 1960s by Carl A. Petri in the context of computer systems (however, he considered such nets much more earlier as 
a tool for modeling chemical reactions) [6]. For years they have been studied in the context of technical systems but during the last years they are tried to be used to describe and analyze biological phenomena, especially metabolic, transduction and gene regulatory pathways and networks. Petri nets, on one hand, have intuitive graphical representation, and on the other, their properties can be analyzed using formal methods. Moreover, many freely available software tools support this analysis and allow for simulation of nets behavior.

The models expressed in the language of Petri net theory are qualitative. As mentioned before, in the case of modeling biological systems this property is helpful since the availability of quantitative biological data is usually very limited. Moreover, the Petri net based model describes the structure of a biological system which is, most often, fundamental for its behavior. So, the model of this type contains a lot of information about the described biological phenomena crucial for their nature.

It should be also mentioned that in the case of building a formal model of a biological system often is a challenging task. It follows from several facts. One of them is that such systems are usually very complex and that they are not developed by humans. So, its complex structure has to be discovered. Another reason of the difficulty is the fact that the knowledge concerning the modeled system is usually rather imprecise since it is expressed in an informal way. In addition, it is distributed among many publications, since usually the subprocesses being parts of the system are described in the literature as separate biological entities. Moreover, not very rarely are cases where some contradicting statements and hypotheses concerning some parts of the modeled systems are formulated and published. And, finally, the knowledge is usually incomplete and some open questions should be answered during the process of developing the model.

The Petri nets in their classical version can be used for building the qualitative model of the studied biological system. But since the nets had been defined many extensions of them have been proposed. Most of them allows for including qualitative information of various types into the model what makes Petri nets very flexible and powerful mathematical tool. Indeed, usually the structure of the net can remain unchanged when the qualitative information is added. Moreover, some variants of Petri nets, from formal point of view, are equivalent to systems of differential equations.

The possibility of including quantitative information into the Petri net based models of biological systems is important since in such systems time dependencies among processes and amounts of the substances required for the execution of them and produced as their results are quite important. As has been said before, even the information about the structure of the biological system allows for interesting analyses of its properties, but if the qualitative data are available they can considerably improve the quality of the model.

Atherosclerosis is the major source of morbidity and mortality in the Western world. Growing evidence indicates that chronic and acute overproduction of reactive oxygen species (ROS) under pathophysiologic conditions is integral in the development of atherosclerotic plaque. ROS mediate various signaling pathways that underlie vascular inflammation in atherogenesis from the initiation of fatty streak development through lesion progression to ultimate plaque rupture. Plaque rupture and thrombosis 
result in the acute clinical complications of myocardial infarction and stroke. Many data support the notion that ROS have a crucial role in atherosclerosis and other vascular diseases. Moreover, oxidative modifications in the arterial wall can contribute to the arteriosclerosis when the balance between oxidants and antioxidants shifts in favour of the former. Although much is known about the role of oxidative stress in atherosclerosis, the process is not fully understood [10].

In this paper a Petri net based model of oxidative stress in atherosclerosis is presented and analyzed. In the second section an informal description of the modeled process is provided. In the third section Petri nets and some of their basic properties especially useful for the analysis of biological system are formally described. In the fourth section the Petri net based model of the oxidative stress in atherosclerosis is presented. The paper ends with conclusions given in the fifth section.

\section{The oxidative stress in atherosclerosis}

Gone are the days when arteriosclerosis was seen merely as a process in which lipid accumulation occurs in the walls of blood vessels. There is currently no doubt that both the induction and progression of atherosclerosic plaque formation are complex processes involving numerous factors coupled with each other. The most important of these include high blood pressure, cholesterol, increased production of free radicals, inflammation, endothelial damage, decreased levels of HDL cholesterol and increased prothrombotic activity.

According to the unified theory of the formation of atherosclerosis, its essence are disturbances in the complex reactions occurring between vascular wall cells, blood cells and plasma lipoproteins. They lead to endothelial dysfunction, platelets activation, oxidation and aggregation of lipoproteins, macrophages transformation in foam cells, inflammation and coagulation.

Under the influence of the inflammatory process comes to changes in the endothelial activation and increased expression of many proteins, including for example the vascular cell adhesion molecule-1 (VCAM1), which after binding to the very late antigen-4 (VLA4), mediates the adhesion of lymphocytes, monocytes, eosinophils, and basophils to vascular endothelium. After this binding it comes to early leukocytes recruitment to the nascent atherosclerotic lesions. Then early leukocytes, as the monocytes, penetrate intima media (the inner part of the arterial wall). The chemokine (C-C motif) receptor 2 (CCR2), and its principle ligand: monocyte chemoattractant protein-1 (MCP1) attracting monocytes from the vessel lumen into the subendothelial space have been well documented for their ability to induce monocyte infiltration [12]. Thus, under their influence monocytes penetrate intima media and finally mature into different types of macrophages, being stimulated by macrophage colony stimulating factor (M-CSF). M-CSF functions as a chemotactic factor for monocytes, regulates the effector functions of mature monocytes and macrophages, and modulates inflammatory responses by stimulating the production of other cytokines and growth factors.

Recruitment, activation, survival, and proliferation of mononuclear phagocytes 
in the vessel wall contribute importantly to atherosclerosis progression. Afterwards, macrophages in the subendothelial space, by using scavenger receptors, capture lipoproteins which are modified by glycation or peroxydation processes, what finally leads to their transformation into the foam cells. This transformation mainly depends on integral membrane protein cluster of differentiation 36 (CD36) and macrophage scavenger receptors class A (SRA). Some of the foam cells undergo apoptosis which in turn leads to endothelial dysfunction and atherosclerotic plaque area enlargement The rest of the foam cells begins to synthesize many substances, such as the growth factors, cytokines and matrix metalloproteinases (MMPs) that contribute to the intensification of the local process of atherosclerosis. MMPs are extracellular matrix degrading enzymes with plaque destabilisating characteristics. They contribute to a reduction in fibrous cap which in turn leads to plaque rupture and the emergence of full-blown atherosclerosis. Thus, their serum levels may predict coronary atherosclerosis in humans [4]. In addition, it comes to intensity of atherosclerosis through the synthesis of numerous cytokines that stimulate the endothelium to synthesize a number of substances, such as selectin E, VCAM1 and intercellular adhesion molecule-1 (ICAM1), that attract monocytes to developing new plaque.

In the development of atherosclerosis very important role play oxdative stress, which lead to lypoproteins modification. Low-density lipoproteins (LDL) react with unstable free radicals and are modified into the oxidized form (ox-LDL). The majority of free radicals that damage biological systems are oxygen free radicals, more generally known as ROS, such as the hydroxyl radical $(\bullet \mathrm{OH})$, hydrogen peroxide $\left(\mathrm{H}_{2} \mathrm{O}_{2}\right)$, or superoxide anion $\left(\mathrm{O}_{2}^{\bullet-}\right)$. The contribution of iron in lipids peroxidation process is unquestionable. It has the ability to participate in the redox reactions. The free iron is noxious to cells because it catalyzes the generation of hydroxyl radicals $(\bullet \mathrm{OH})$ from superoxide $\left(\mathrm{O}^{\bullet-}\right)$ and hydrogen peroxide $\left(\mathrm{H}_{2} \mathrm{O}_{2}\right)$ via the Fenton reaction. For more detailed information see in [2].

The reaction of nitric oxide (NO) with superoxide $\mathrm{O}_{2}^{\bullet-}$ to form the much more powerful oxidant peroxynitrite $\left(\mathrm{ONOO}^{-}\right)$is a key element in resolving the contrasting roles of NO in physiology and pathology. The increased superoxide production and increased NO from inducible nitric oxide synthase (iNOS) overexpression favor the generation of peroxynitrite. The formation of free radicals increases degranulation of mast cells, which in turn are responsible for the synthesis of cytokines activating iNOS, which increases NO production.

Presented selected aspects of the impact of oxidative stress and inflammation on atherosclerotic plaque formation show how complex are the processes and reveal the network of links between them.

\section{Petri nets}

In this section a short introduction to the theory of Petri nets is given. Some basic definitions concerning the structure and behavior of these nets are provided. Moreover, some properties of such nets are defined and methods of analysis especially important in the context of biological systems are described. 
A Petri net structure is based on a directed bipartite graph. In such a graph there are two disjoint sets of vertices, called in the case of these nets places and transitions. Places correspond to some passive elements of the modeled systems while transitions correspond to their active components. In case of biological systems transitions may model, for example, chemical reactions and places correspond to the compounds necessary for the reactions to take place or to the products of these reactions. Every arc in the net has to be incident with two vertices of different types (as in every bipartite graph) modeling casual relations between the passive and the active system components.

In graphical representation of a Petri net places are denoted as circles, transitions as rectangles and arcs as arrows. Each arc is labeled by a positive integer number called weight (and in a graphical representation usually only weights greater than one are shown).

Formally, a Petri net can be defined as follows [5]:

\section{DEFINITION}

A Petri net is a 5 -tuple $Q=\left(P, T, F, W, M_{0}\right)$, where:

$P=\left\{p_{1}, p_{2}, \ldots, p_{n}\right\}$ is a set of places,

$T=\left\{t_{1}, t_{2}, \ldots, t_{m}\right\}$ is a set of transitions,

$F \subseteq(P \times T) \cup(T \times P)$ is a set of arcs,

$W: F \rightarrow \mathbb{Z}^{+}$is a weight function,

$M_{0}: P \rightarrow \mathbb{N}$ is an initial marking,

$P \cap T=\emptyset \wedge P \cup T \neq \emptyset$.

For each transition $t_{j}$ there is a set of input places $p_{i}$, i.e. the places being starting vertices of arcs whose ending vetex is $t_{j}$ - the places for which $\operatorname{arc}\left(p_{i}, t_{j}\right)$ exists in the net and a set of output places $p_{k}$, i.e. places being ending vertices of arcs whose starting vertex is transition $t_{j}$ - the places for which $\operatorname{arc}\left(t_{j}, p_{k}\right)$ exists. For some transitions the set of input places is empty - they are called input transitions. There are also transitions for which the set of output places is empty - they are called output transitions. Input and output transitions may correspond to some connections of the modeled system with its environment.

Places, transitions and arcs define the structure of a Petri net, but one of the fundamental properties of such nets is dynamics brought to the net by tokens residing in places. They correspond to amounts of passive system components and flow through a transition from its input places to its output places. In graphical representation of a Petri net tokens are represented as dots or numbers.

The distribution of tokens over places of the Petri net correspond to the state of the modeled system and is called marking. The initial marking describes the initial system state. A marking can be changed when the token flows through transition what takes place according to the following transition activation rule [5]:

1) transition $t_{j}$ is enabled if in every of its input places $p_{i}$ there is a number of tokens greater than or equal to $w\left(p_{i}, t_{j}\right)$, where $w\left(p_{i}, t_{j}\right)$ is a weight of $\operatorname{arc}\left(p_{i}, t_{j}\right)$;

2) an enabled transition can be fired;

3) firing of transition $t_{j}$ removes $w\left(p_{i}, t_{j}\right)$ tokens from each of its input places $p_{i}$ and 
adds $w\left(t_{j}, p_{k}\right)$ tokens for each of its output places $p_{k}$.

Every input transition is continuously enabled and firing every output transition does not produce any tokens.

The graphical representation of a Petri net is very intuitive and useful for simulation of the net behavior but of limited value for an analysis of the formal properties of the net. Hence, another representation, called an incidence matrix is used for this purpose. In such a matrix $A=\left(a_{i j}\right)_{n \times m}$ the rows correspond to places and the columns correspond to transitions. Each entry $a_{i j}$ of the matrix is an integer number equal to a difference between the numbers of tokens residing in place $p_{i}$ before and after firing transition $t_{j}$.

There are a number of structural properties of a Petri net which can be important for the properties of the modeled system. Among others there are:

A Petri net is ordinary if a weight of every arc is equal to one.

A Petri net is homogenous if for every place $p_{i}$ all arcs whose starting point is $p_{i}$ has the same weight.

A Petri net is pure if there are no loops in the net, i.e. if it does not contain read arcs.

A Petri net is conservative if for each transition $t_{j}$ the sum of weights of its ingoing arcs (i.e. the ones of the form $\left(p_{i}, t_{j}\right)$ for some $\left.p_{i}\right)$ is equal to the sum of the weights of the outgoing arcs (i.e. the arcs of the form $\left(t_{j}, p_{k}\right)$ for some $\left.p_{k}\right)$.

A Petri net is connected if from each vertex of the net there exists an undirected path to every other vertex of the net.

A Petri net is strongly connected if from every vertex of the net there exists a directed path to any other vertex of the net.

A Petri net is bounded if in every possible marking there is not more than $k$ tokens in any place of the net for some integer number $k$.

A Petri net is structurally bonded if it is bounded for every initial marking.

A Petri net is structurally conflict free if there are no two transitions which have the same input place.

In a Petri net a dead state is reachable if a marking of the net is possible for which no transition can fire.

A Petri net has anon-blocking multiplicity if for every place of the net the minimal weight of its ingoing arcs is greater than or equal to the maximal weight of its outgoing arcs.

For Petri nets being models of biological systems especially important is an analysis of their properties related to invariants. The invariants correspond to some parts of the net which can be identified with elementary functional units of the modeled system when the model is properly constructed. There are two kinds of invariants, i.e. $p$ invariants (place invariants) and t-invariants (transition invariants). A p-invariant is a vector $y \in \mathbb{N}^{n}$ satisfying the equation $[5,8,7]$ :

$$
y \cdot A=0
$$

A p-invariant corresponds to a set of places over which a weighted sum of tokens is constant. In the context of biological systems a p-invariant corresponds to a subsystem for which an amount of substances is constant. In order to influence the behavior of 
the net a p-invariant should contain at least one token in an initial marking.

A support $\operatorname{supp}(y)$ of invariant $y$ is a set of places corresponding to the non-zero entries of $y$, i.e. $\operatorname{supp}(y)=\left\{p_{i}: y_{i}>0\right\}$. p-invariant $y$ is minimal if there is no other p-invariant $y^{\prime}$ such that $\operatorname{supp}\left(y^{\prime}\right) \subset \operatorname{supp}(y)$. Since any p-invariant can be obtained as a linear combination of minimal p-invariants it is sufficient to consider only the minimal ones. If every place of the net is contained in support of some p-invariant the net is covered by p-invariants. In such a net a weighted sum of tokens is constant.

Let $I_{p}$ be the set of p-invariants of a Petri net and $I_{p}^{(\min )}$ be the set of all minimal p-invariants of this net, i.e.

$$
\begin{gathered}
I_{p}=\left\{y \in \mathbb{N}^{n}: y \cdot A=0\right\} \\
I_{p}^{(\min )}=\left\{y \in I_{p}: \forall_{y^{\prime} \in I_{p} \backslash\{y\}} \operatorname{supp}\left(y^{\prime}\right) \not \subset \operatorname{supp}(y)\right\}
\end{gathered}
$$

A $t$-invariant is vector $x \in \mathbb{N}^{m}$ satisfying the equation $[5,8,7]$ :

$$
A \cdot x=0
$$

Analogously like in the case of p-invariants, support $\operatorname{supp}(x)$ of t-invariant $x$ is a set of transitions which correspond to the non-zero entries of $x$, i.e. $\operatorname{supp}(x)=\left\{t_{j}: x_{j}>0\right\}$. Minimal t-invariant $x$ is such an invariant for which there is no other t-invariant $x^{\prime}$ such that $\operatorname{supp}\left(x^{\prime}\right) \subset \operatorname{supp}(x)$. Also in the case of t-invariants each of them can be obtained as a sum of the minimal ones, so considering only the minimal ones is sufficient. A Petri net should be covered by t-invariants (i.e. each transition should be an element of a support of some t-invariant). In such a case each transition influences the behavior of the net.

Let $I_{t}$ be the set of t-invariants of a Petri net and $I_{t}^{(\min )}$ be the set of all minimal t-invariants i.e.

$$
\begin{gathered}
I_{t}=\left\{x \in \mathbb{N}^{m}: A \cdot x=0\right\} \\
I_{t}^{(\min )}=\left\{x \in I_{t}: \forall_{x^{\prime} \in I_{t} \backslash\{x\}} \operatorname{supp}\left(x^{\prime}\right) \not \subset \operatorname{supp}(x)\right\}
\end{gathered}
$$

In case of Petri net based models of biological systems especially interesting are feasible t-invariants $[9,8,7]$. A minimal t-invariant is feasible if all transitions being elements of its support can be fired without firing any other transition. Let $I_{t}^{(f)}$ denote the set of feasible t-invariants of Petri net. The reason that a minimal t-invariant is not a feasible one is an occurrence of read arcs in the net. If a transition is connected via an arc of this type with its input place firing of this transition does not remove any tokens from this place. From this follows that read arcs are not reflected in the incidence matrix. In the models of biological systems arcs of this type can be used to model chemical reactions activated by catalysts, which are necessary to start the reaction but are not consumed.

When a Petri net based model is a basis for the analysis of the biological system the properties of the net should be correlated with the properties of the system. In particular, every minimal t-invariant should be correlated with some functional subunit of the biological system. However, in many cases the number of such invariants is so large that instead of analyzing biological meaning of each of them some groups of feasible t-invariants or transitions are analyzed. 
Transitions can be grouped into the so-called MCT sets (Maximal Common Transition sets). They contain transitions belonging to supports of exactly the same feasible t-invariants. These sets correspond to a partition of the biological system into disjoint subunits whose biological meaning should be determined.

Let $I_{t}^{(f)}\left(t_{j}\right)$ be a set of all feasible t-invariants of a Petri net which share transition $t_{j} \in T$. We can define the set of all MCT sets $Z_{M C T}$ in the following way:

$$
\begin{aligned}
& I_{t}^{(f)}\left(t_{j}\right)=\left\{x \in I_{t}^{(f)}: t_{j} \in \operatorname{supp}(x)\right\} \\
& Z_{M C T}=\left\{m \subseteq T: \forall_{t_{1}, t_{2} \in m, t_{3} \in T \backslash m} I_{t}^{(f)}\left(t_{1}\right)=I_{t}^{(f)}\left(t_{2}\right) \neq I_{t}^{(f)}\left(t_{3}\right)\right\}
\end{aligned}
$$

Another method of the analysis of Petri net based model properties in the case where the number of feasible t-invariants is large is based on t-clusters. They are groups of feasible t-invariants which are similar to each other according to some measure of similarity. Here, Tanimoto coefficient is often used. According to this coefficient similarity $s\left(x_{i}, x_{j}\right)$ of t-invariants $x_{i}$ and $x_{j}$ is equal to

$$
s\left(x_{i}, x_{j}\right)=\frac{\operatorname{supp}\left(x_{i}\right) \cap \operatorname{supp}\left(x_{j}\right)}{\operatorname{supp}\left(x_{i}\right) \cup \operatorname{supp}\left(x_{j}\right)}
$$

Distance $d_{i j}$ between $x_{i}$ and $x_{j}$ is defined as

$$
d_{i j}=1-s\left(x_{i}, x_{j}\right)
$$

Another measure of similarity used to define t-clusters is Pearson correlation coefficient. In this case similarity of t-invariants $x_{i}$ and $x_{j}$ is equal to Pearson correlation coefficient $r\left(x_{i}, x_{j}\right)$, i.e.

$$
s\left(x_{i}, x_{j}\right)=r\left(x_{i}, x_{j}\right)
$$

Since Pearson correlation coefficient $\mathrm{R}$ takes values in the range $[-1,1]$, distance takes values in the range $[0,2]$. Distances $d_{i j}$ are elements of matrix $D$ which is a basis for grouping t-invariants into t-clusters. For determining these clusters standard algorithms, as UPGMA, can be used. In each step two objects (i.e. t-invariants or clusters) whose distance is minimal are joint into a new cluster. In this way a dendrogram whose leaves are t-invariants is constructed. The procedure stops when a single cluster is obtained. The resulting t-clusters can be determined by taking into account an upper part of the dendrogram, i.e. the one between the root and some chosen distance level (threshold) $\lambda$ ( $\lambda$ has the same domain like the chosen distance measure, e.g. $\lambda \in[0,2]$ for the case of Pearson correlation coefficient). The dendrogram nodes located at this level correspond to the calculated t-clusters. Let us denote the set of t-clusters of a Petri net obtained for distance level $\lambda$ (using a particular clustering method) by $C(\lambda)$.

\section{The model}

Biochemical facts described in section 2 were assembled into a Petri net based model. This model contains relations between states (places) and their transformations (transitions). States represent both biochemical and biological entities. An example of a 
biochemical entity is place $p_{22}$ (iron ions $F e^{3+}$ ) while place $p_{0}$ (inflammed endothelium) represents a biological state. Transformations are represented similarly, e.g. $t_{29}$ ( $F e$ reduction reaction) is a biochemical transformation while $t_{47}$ (atheroslerosis progression) is a biological one.

The model is shown in Figure 2. It contains 43 places $\left(P=\left\{p_{0}, p_{1}, \ldots, p_{42}\right\}\right)$ and 51 transitions $\left(T=\left\{t_{0}, t_{1}, \ldots, t_{50}\right\}\right)$. The names and the biological meaning of the places and the transitions are described in Tables 2 and 3.

\subsection{Structural analysis}

The presented model is a discrete Petri net where weights of all arcs are equal to 1 . It does not contain information about reaction speed and concentrations of biochemical entities and thus cannot provide information about quantitative relations between its states.

However, structural analysis can provide some information about properties of the model. Since it contains only arcs which are weighted with one, it is ordinary and homogeneous. As mentioned above, such models concentrate on information processing rather than on quantitative analysis. Table 1 presents structural properties of the model with descriptions.

\subsection{Invariant analysis}

The model contains two minimal p-invariants $\left(I_{p}^{(\min )}=\left\{y_{1}, y_{2}\right\}\right)$ listed in Table 4 which represent two cyclic transformations, i.e. $\mathrm{Fe}^{2+} / \mathrm{Fe}^{3+}$ and citruline/L-arginine. The initial marking provides tokens in these p-invariants, so they may contribute to the behavior of the model.

The model is covered by 42 minimal t-invariants $\left(I_{t}^{(\min )}=\left\{x_{1}, x_{2}, \ldots, x_{42}\right\}\right.$ which are listed in Table 6 ). Since the model does not contain read arcs the minimal t-invariants are also feasible t-invariants. Then induced $10 \mathrm{MCT}$ sets $\left(Z_{M C T}=\right.$ $\left.\left\{m_{1}, m_{2}, \ldots, m_{10}\right\}\right)$ listed in Table 5 . Table 6 presents the minimal t-invariants as a sum of MCT-sets and single transitions.

The t-invariant coverage is an important property which validates the model. A valid biological model has to keep homeostasis, so it has to be either bounded or covered by t-invariants. The model which is bounded may represent thermodynamically closed processes, while biological models are thermodynamically open. Presented model is not bounded as mentioned in Table 1. When the net is covered by t-invariants it means that each transition is a part of some token flow in the net and contribute to its behavior. If the net was not covered by t-invariants there can be some places which accumulate tokens to the infinity (and thus such a net is not able to keep homeostasis).

Each t-invariant represents some biological process. These processes can be grouped into clusters because there are usually many common subpaths shared by them - such subpaths may be interesting from biological point of view. Using clustering method 
and distance measure described in the previous section we obtained 6 t-clusters for chosen similarity threshold $\lambda=0.3$ (i.e. $C(0.3)=\left\{c_{1}, c_{2}, \ldots, c_{6}\right\}$. These clusters are listed in Table 6 . In the table the t-invariants are sorted according to distance measure, so clusters are presented as continuous range of t-invariants (e.g. cluster $c_{1}$ contains t-invariants range $x_{1}-x_{27}$ ). Biological descriptions of these clusters can be found in Table 7 .

The similarity threshold $\lambda=0.3$ was chosen manually according to the possible biological meaning of induced clusters and observed strong separation of large clusters $c_{1}$ and $c_{2}$ from the other ones. The distance between two sets of clusters $\left\{c_{1}, c_{2}\right\}$ and $\left\{c_{3}, c_{4}, c_{5}, c_{6}\right\}$ is close to 1 , what means there is no correlation between these sets. Clusters $c_{1}$ and $c_{2}$ are based on the largest MCT set $m_{1}$, which contains transitions responsible for immune system response.

Clusters $c_{1}$ and $c_{2}$ differ mainly in the way how iNOS is obtained. For cluster $c_{1}$ iNOS is obtained from peroxynitrite anion $\left(\mathrm{OONO}^{-}\right)$activated by cytokines $\left(t_{42}\right)$, while for $c_{2}$ it is obtained from mast cells degranulation $\left(t_{41}\right)$. Except of t-invariants $x_{24}$ and $x_{25}$ cluster $c_{1}$ does not contain t-invariants based on MCT set $m_{10}$ which represents atherosclerosis progression, while whole cluster $c_{2}$ depends on $m_{10}$. This means that mast cells degranulation is strongly correlated with process of atherosclerosis progression.

The other clusters represent groups of processes which are not based on immune system response. Clusters $c_{3}, c_{4}, c_{5}$ influence atherosclerosis progression (each t-invariant is based on $m_{10}$ ). They influence atherosclerosis progression in the following ways:

- $c_{3}$ - is based on transition $t_{15}$ - it represents rich cholesterol diet which causes expression of adhesion protein VCAM1,

- $c_{4}$ - is based on transition $t_{36}$ - it represents lipids peroxidation by peroxynitrite which leads to expression of protein ICAM1,

- $c_{5}$ - is based on MCT set $m_{3}$ which represents expression of adhesion protein VCAM1 caused by inflammatory process.

Clusters $c_{3}, c_{4}, c_{5}$ group processes which are not related to immune system response and lead to expression of proteins ICAM1 or VCAM1 which influence atherosclerosis progression.

The last cluster $c_{6}$ represents endothelium activation influenced by $\mathrm{NO}$ and does not influence atherosclerosis progression.

\section{Conclusions}

In this paper the Petri net based model of oxidative stress in atherosclerosis has been presented and analyzed. The analysis has been mainly based on t-invariants since they are especially important in the context of biological systems. The biological meaning of each of them has been determined and moreover, MCT-sets and t-clusters 
have been calculated and analyzed. The presented model is a qualitative one, so it does not contain any quantitative information, e.g. concerning substance concentrations or time dependencies. However, it provides important information about the structure of the analyzed system which is crucial for the nature and behavior of the modeled biological phenomenon. On the other hand, the model can be relatively easily complemented by quantitative data if they will be available.

Atherosclerosis is a very complex not fully understood process and the presented model describes some of its aspects. One of the observations made on the basis of the model analysis is that mast cells degranulation in immune system response is strongly correlated with atherosclerosis progression, what confirms some hypotheses.

The model is a starting point for future research whose goal is to support the understanding of the complex process of atherosclerosis development by the construction and the analysis of exact mathematical models of this biological phenomenon.

\section{References}

[1] Bertalanffy L., General System Theory. Foundations, Development, Applications, George Braziller, Inc., New York, 1969.

[2] Formanowicz D., Do changes in iron metabolism contribute to the acceleration of the atherosclerosis process?, Biotechnologia, 92 , 2011, 180-192.

[3] Klipp E., Liebermeister W., Wierling C., Kowald A., Lehrach H., Herwig R., Systems Biology. A Textbook, Wiley-VCH, Weinheim, 2009.

[4] Lehrke M., Greif M., Broedl U.C., Lebherz C., Laubender R.P., Becker A., Ziegler F., Tittus J., Reiser M., Beckerm C., Gke B., Steinbeck G., Leber A.W., Parhofer K.G., MMP-1 serum levels predict coronary atherosclerosis in humans, Cardiovascular Diabetology, 8, 2009, 50.

[5] Murata T., Petri nets: Properties, analysis and applications, Proceedings of the IEEE, 77, 1989, 541-580.

[6] Petri C.A., Kommunikation mit automaten, Institut für Instrumentelle Mathematik, Bonn, 1962.

[7] Sackmann A., Discrete modeling, in: I. Koch., W. Reisig, F. Schreiber, (eds.) Modeling in Systems Biology. The Petri Net Approach, Springer London, 2011, $59-72$.

[8] Sackmann A., Formanowicz D., Formanowicz P., Koch I., Błażewicz J., An analysis of the Petri net based model of the human body homeostasis process, Computational Biology and Chemistry, 31, 2007, 1-10.

[9] Sackmann A., Heiner M., Koch I., Application of Petri net based analysis techniques to signal transduction pathway, BMC Bioinformatics, 7, 2006, 482. 
[10] Stocker R., Keaney J.F., Jr. Role of Oxidative Modifications in Atherosclerosis. Physiol Rev, 84, 2004, 1381-1478.

[11] Szallasi Z., Stelling J., Periwal V., System modeling in cellular biology: From concepts to nuts and bolts, The MIT Press, Cambridge, 2006.

[12] Tylaska L.A., Boring L., Weng W., Aiello R., Charo I.F., Rollins B.J., Gladue R.P., CCR2 regulates the level of MCP-1/CCL2 in vitro and at inflammatory sites and controls t cell activation in response to alloantigen, Cytokine, 18, 2002, 184-190.

[13] Wiener N., Cybernetics or Control and Communication in the Animal and the Machine, 2nd edition, The Technology Press (MIT)/John Wiley \& Sons, New York, London, 1961.

Received February, 2012 
Table 1: Structural properties of the model

\begin{tabular}{|c|c|c|}
\hline Property & Has property? & Description \\
\hline ordinary & yes & weight of each arc is equal to one \\
\hline homogeneous & yes & $\begin{array}{l}\text { for each place the set of outcoming arcs } \\
\text { consists of arcs of the same weight }\end{array}$ \\
\hline pure & yes & does not contain loops (read arcs) \\
\hline $\begin{array}{l}\text { non-blocking multi- } \\
\text { plicity }\end{array}$ & yes & $\begin{array}{l}\text { this is immediate property of ordinary net } \\
\text { (if each arc has weight equal to one, then } \\
\text { for each place its minimal weight of ingo- } \\
\text { ing arcs is greater or equal to the maximal } \\
\text { weight of its outgoing arcs) }\end{array}$ \\
\hline conservative & no & $\begin{array}{l}\text { there are transitions which have different } \\
\text { sum of weights of ingoing and outgoing arcs } \\
\text { (in ordinary net it is equivalent to different } \\
\text { number of ingoing and outgoing arcs) - e.g. } \\
t_{44}\end{array}$ \\
\hline $\begin{array}{l}\text { structurally } \\
\text { conflict-free }\end{array}$ & no & $\begin{array}{l}\text { there are transitions which share the same } \\
\text { preceding place }\left(\text { e.g. } t_{2} \text { and } t_{20}\right)\end{array}$ \\
\hline connected & yes & $\begin{array}{l}\text { there are no places/transitions which have } \\
\text { no influence on the model (there exists } \\
\text { undirected path between each pair of ver- } \\
\text { tices) }\end{array}$ \\
\hline strongly connected & no & $\begin{array}{l}\text { states are not fully reversible (there is no } \\
\text { directed path between each pair of vertices) }\end{array}$ \\
\hline bounded & no & $\begin{array}{l}\text { the model is connected with 'outer world' } \\
\text { via initial and final transitions - initial } \\
\text { transitions provide tokens, so there is no } \\
\text { limit on number of tokens in the net }\end{array}$ \\
\hline $\begin{array}{l}\text { structurally } \\
\text { bounded }\end{array}$ & no & $\begin{array}{l}\text { if the net is not bounded it cannot be struc- } \\
\text { turally bounded as well }\end{array}$ \\
\hline p-invariants & yes & $\begin{array}{l}\text { there are } 2 \text { minimal p-invariants }\left(y_{1}, y_{2}\right. \\
\text { listed in table } 4)\end{array}$ \\
\hline $\begin{array}{l}\text { covered by } \quad \text { - } \\
\text { invariants }\end{array}$ & no & $\begin{array}{l}\text { there are only } 4 \text { places in p-invariants - } \\
\text { in general net cannot be covered by p- } \\
\text { invariants if it is not bounded }\end{array}$ \\
\hline t-invariants & yes & $\begin{array}{l}\text { there are } 42 \text { minimal t-invariants }\left(x_{1}-x_{42}\right. \\
\text { listed in table } 6)\end{array}$ \\
\hline $\begin{array}{l}\text { covered by t- } \\
\text { invariants }\end{array}$ & yes & each transition is a part of some t-invariant \\
\hline deadlock-trap & yes & $\begin{array}{l}\text { there are no structural traps apart from } \\
\text { existing two p-invariants - these two p- } \\
\text { invariants have non-zero initial marking, so } \\
\text { the model has no deadlocks }\end{array}$ \\
\hline $\begin{array}{l}\text { dead state reach- } \\
\text { able }\end{array}$ & no & $\begin{array}{l}\text { there is no such marking where no transi- } \\
\text { tion can fire }\end{array}$ \\
\hline
\end{tabular}




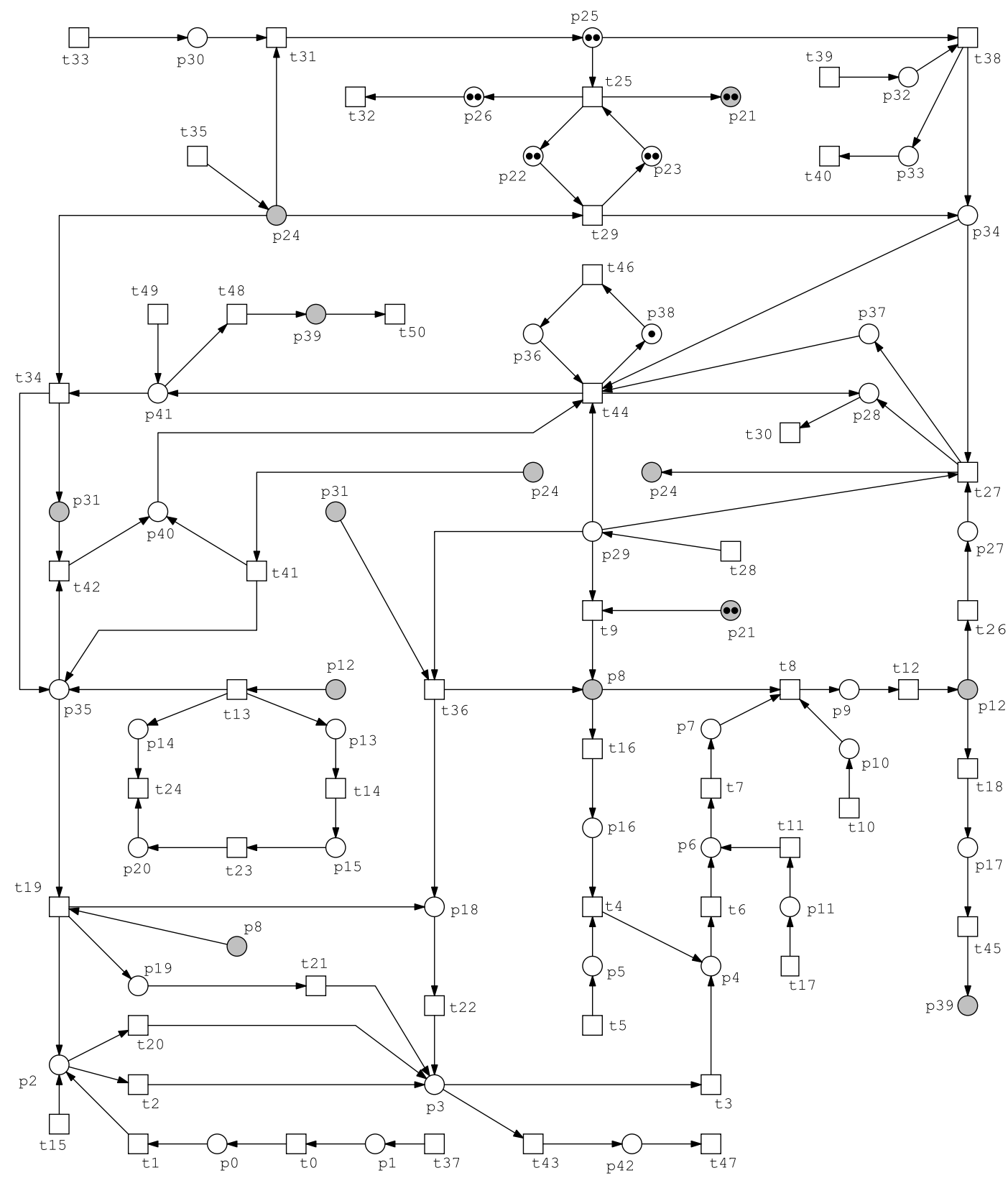

Figure 1: The Petri net based model 
Table 2: The IDs and names of the places of the model

\begin{tabular}{|l|l|l|l|}
\hline ID & Place name & ID & Place name \\
\hline$p_{0}$ & inflammed endothelium & $p_{22}$ & iron ions $F e^{3+}$ \\
\hline$p_{1}$ & inflammation & $p_{23}$ & iron ions $F e^{2+}$ \\
\hline$p_{2}$ & VCAM1 & $p_{24}$ & superoxide anion \\
\hline$p_{3}$ & $\begin{array}{l}\text { early leukocytes in inflammed } \\
\text { endothelium }\end{array}$ & $p_{25}$ & hydrogen peroxide \\
\hline$p_{4}$ & monocytes in intima media & $p_{26}$ & hydroxyl ion \\
\hline$p_{5}$ & CCR2 & $p_{27}$ & $\begin{array}{l}\text { newly formed NADPH oxidase } \\
\text { enzyme complex }\end{array}$ \\
\hline$p_{6}$ & intimal macrophages & $p_{28}$ & NADP cation \\
\hline$p_{7}$ & $\begin{array}{l}\text { macrophages with scavenger re- } \\
\text { ceptors SRA or CD36 }\end{array}$ & $p_{29}$ & NADPH \\
\hline$p_{8}$ & $\begin{array}{l}\text { lipoprotein particles modified by } \\
\text { oxidation }\end{array}$ & $p_{30}$ & SOD \\
\hline$p_{9}$ & $\begin{array}{l}\text { scavenger receptors/modified } \\
\text { lipoprotein particles complex }\end{array}$ & $p_{31}$ & peroxynitrite anion (OONO $\left.{ }^{-}\right)$ \\
\hline$p_{10}$ & $\begin{array}{l}\text { lipoprotein particles modified by } \\
\text { glycation }\end{array}$ & $p_{32}$ & catalase \\
\hline$p_{11}$ & M-CSF & $p_{33}$ & water \\
\hline$p_{12}$ & macrophage foam cell & $p_{34}$ & oxygen \\
\hline$p_{13}$ & MMPs & $p_{35}$ & cytokines \\
\hline$p_{14}$ & tissue factors & $p_{36}$ & L-arginine \\
\hline$p_{15}$ & plaque fibrous cap reduction & $p_{37}$ & hydrogen cation \\
\hline$p_{16}$ & MCP1 & $p_{38}$ & citruline \\
\hline$p_{17}$ & apoptotic bodies & $p_{39}$ & activated endothelium \\
\hline$p_{18}$ & ICAM1 & nitric oxide \\
\hline$p_{19}$ & selectin E & atherosclerotic plaque \\
\hline$p_{20}$ & plaque rupture & \\
\hline$p_{21}$ & highly toxic hydroxyl radical & \\
\hline
\end{tabular}


Table 3: The IDs and names of the transitions of the model

\begin{tabular}{|c|c|c|c|}
\hline ID & Transition name & ID & Transition name \\
\hline$t_{0}$ & $\begin{array}{l}\text { endothelial cells activation by in- } \\
\text { flammation }\end{array}$ & $t_{26}$ & $\begin{array}{l}\text { cytostolic components translo- } \\
\text { cate to the membrane }\end{array}$ \\
\hline$t_{1}$ & $\begin{array}{l}\text { increased expression leukocyte } \\
\text { adhesion molecules }\end{array}$ & $t_{27}$ & $\begin{array}{l}\text { processes catalysed by NADPH } \\
\text { oxidase }\end{array}$ \\
\hline$t_{2}$ & $\begin{array}{l}\text { early leukocytes recruitment to } \\
\text { the nascent atherosclerotic lesion } \\
\text { VCAM1/VLA4 binding }\end{array}$ & $t_{28}$ & NADPH synthesis \\
\hline$t_{3}$ & $\begin{array}{l}\text { monocytes penetrate intima me- } \\
\text { dia }\end{array}$ & $t_{29}$ & Fe reduction reaction \\
\hline$t_{4}$ & MCP1/CCR2 binding & $t_{30}$ & NADP cation usage \\
\hline$t_{5}$ & CCR2 expression & $t_{31}$ & reaction catalyzed by SOD \\
\hline$t_{6}$ & $\begin{array}{l}\text { monocytes acquires characteris- } \\
\text { tics of tissue macrophages }\end{array}$ & $t_{32}$ & hydroxyl ion usage \\
\hline$t_{7}$ & scavanger receptors expression & $t_{33}$ & activation of defense mechanisms \\
\hline$t_{8}$ & $\begin{array}{l}\text { scavanger receptors modyfied } \\
\text { lipoprotein particles binding }\end{array}$ & $t_{34}$ & reaction catalyzed by $\mathrm{NO}$ \\
\hline$t_{9}$ & $\begin{array}{l}\text { lipids peroxidation by hydroxyl } \\
\text { radical }\end{array}$ & $t_{35}$ & superoxide anion synthesis \\
\hline$t_{10}$ & glycation process & $t_{36}$ & $\begin{array}{l}\text { lipids peroxidation by peroxyni- } \\
\text { trite }\end{array}$ \\
\hline$t_{11}$ & activation & $t_{37}$ & inflammatory process \\
\hline$t_{12}$ & transformation & $t_{38}$ & reaction catalyzed by catalase \\
\hline$t_{13}$ & secretion & $t_{39}$ & catalase synthesis \\
\hline$t_{14}$ & extracellular matrix degradation & $t_{40}$ & water usage \\
\hline$t_{15}$ & rich cholesterol diet & $t_{41}$ & mast cells degranulation \\
\hline$t_{16}$ & MCP1 expression & $t_{42}$ & iNOS activation by cytokines \\
\hline$t_{17}$ & $\begin{array}{l}\text { M-CSF synthesis by endotelium } \\
\text { and smooth muscles }\end{array}$ & $t_{43}$ & $\begin{array}{l}\text { atheosclerotic plaque formation } \\
\text { by leukocytes infiltration }\end{array}$ \\
\hline$t_{18}$ & $\begin{array}{l}\text { transformation into apoptotic } \\
\text { bodies }\end{array}$ & $t_{44}$ & reaction catalyzed by iNOS \\
\hline$t_{19}$ & $\begin{array}{l}\text { endotelial stimulation for adhe- } \\
\text { sion proteines expression }\end{array}$ & $t_{45}$ & endothelial injury \\
\hline$t_{20}$ & $\begin{array}{l}\text { monocytes binding to endothe- } \\
\text { lium by VCAM1 }\end{array}$ & $t_{46}$ & L-arginine synthesis \\
\hline$t_{21}$ & $\begin{array}{l}\text { monocytes binding to endothe- } \\
\text { lium by selectin } \mathrm{E}\end{array}$ & $t_{47}$ & atherosclerosis progression \\
\hline$t_{22}$ & $\begin{array}{l}\text { monocytes binding to endothe- } \\
\text { lium by ICAM1 }\end{array}$ & $t_{48}$ & $\begin{array}{l}\text { endothelium activation influ- } \\
\text { enced by NO }\end{array}$ \\
\hline$t_{23}$ & plaque ruptures processes & $t_{49}$ & NO syntheis \\
\hline$t_{24}$ & $\begin{array}{l}\text { clinical symptoms of atheroscle- } \\
\text { rosis }\end{array}$ & $t_{50}$ & $\begin{array}{l}\text { substances synthesis by acti- } \\
\text { vated endothelium }\end{array}$ \\
\hline$t_{25}$ & iron catalyzed Fenton reaction & & \\
\hline
\end{tabular}


Table 4: Place invariants

\begin{tabular}{|l|l|l|}
\hline ID & Places & Description \\
\hline$y_{1}$ & $p_{22}, p_{23}$ & iron ion reduction and oxidation cycle $\left(F e^{2+} / F e^{3+}\right)$ \\
\hline$y_{2}$ & $p_{36}, p_{38}$ & citruline/L-arginine cycle \\
\hline
\end{tabular}

Table 5: The Maximal Common Transition (MCT) sets

\begin{tabular}{|c|c|c|}
\hline ID & Transitions & Description \\
\hline$m_{1}$ & $\begin{array}{l}t_{7}, t_{8}, t_{9}, t_{10}, t_{12}, t_{25}, t_{26}, t_{27}, t_{29}, t_{30} \\
t_{31}, t_{32}, t_{33}, t_{44}, t_{46}\end{array}$ & immune system response \\
\hline$m_{2}$ & $t_{13}, t_{14}, t_{23}, t_{24}$ & $\begin{array}{l}\text { processes leading to clinical } \\
\text { symptoms due to macrophage } \\
\text { foam cells secretion }\end{array}$ \\
\hline$m_{3}$ & $t_{0}, t_{1}, t_{37}$ & $\begin{array}{l}\text { VCAM1 synthesis in endothe- } \\
\text { lium caused by inflammatory } \\
\text { process }\end{array}$ \\
\hline$m_{4}$ & $t_{4}, t_{5}, t_{16}$ & MCP1 binding to CCR2 \\
\hline$m_{5}$ & $t_{19}, t_{21}, t_{22}$ & $\begin{array}{l}\text { growing early leukocytes in in- } \\
\text { flammed endothelium caused by } \\
\text { selectin E and ICAM1 mono- } \\
\text { cytes binding }\end{array}$ \\
\hline$m_{6}$ & $t_{38}, t_{39}, t_{40}$ & $\begin{array}{l}\text { decomposition of hydrogen per- } \\
\text { oxide to water and oxygen }\end{array}$ \\
\hline$m_{7}$ & $t_{11}, t_{17}$ & $\begin{array}{l}\text { growing intimal macrophages } \\
\text { due to M-CSF synthesis }\end{array}$ \\
\hline$m_{8}$ & $t_{18}, t_{45}$ & $\begin{array}{l}\text { growing atheosclerotic plaque } \\
\text { caused by macrophage foam cells }\end{array}$ \\
\hline$m_{9}$ & $t_{28}, t_{35}$ & superoxide anion synthesis \\
\hline$m_{10}$ & $t_{43}, t_{47}$ & $\begin{array}{l}\text { growing atherosclerotic plaque } \\
\text { caused by leukocytes infiltration }\end{array}$ \\
\hline
\end{tabular}


Table 6: t-invariants grouped into t-clusters. Invariants are sorted according to similarity measure applied for the clustering method.

\begin{tabular}{|c|c|c|c|}
\hline ID & MCT sets & Single transitions & t-cluster \\
\hline$x_{1}$ & $m_{1}, m_{4}, m_{9}$ & $t_{6}, t_{34}, t_{42}$ & \multirow{27}{*}{$c_{1}$} \\
\hline$x_{2}$ & $m_{1}, m_{2}, m_{5}, m_{9}$ & $t_{2}, t_{3}, t_{6}, t_{34}, t_{42}$ & \\
\hline$x_{3}$ & $m_{1}, m_{2}, m_{5}, m_{9}$ & $t_{3}, t_{6}, t_{20}, t_{34}, t_{42}$ & \\
\hline$x_{4}$ & $m_{1}, m_{5}, m_{8}, m_{9}$ & $t_{2}, t_{3}, t_{6}, t_{34}, t_{36}, t_{42}, t_{49}, t_{50}$ & \\
\hline$x_{5}$ & $m_{1}, m_{5}, m_{8}, m_{9}$ & $t_{3}, t_{6}, t_{20}, t_{34}, t_{36}, t_{42}, t_{49}, t_{50}$ & \\
\hline$x_{6}$ & $m_{1}, m_{5}, m_{8}, m_{9}$ & $t_{2}, t_{3}, t_{6}, t_{34}, t_{36}, t_{41}, t_{42}, t_{50}$ & \\
\hline$x_{7}$ & $m_{1}, m_{5}, m_{8}, m_{9}$ & $t_{3}, t_{6}, t_{20}, t_{34}, t_{36}, t_{41}, t_{42}, t_{50}$ & \\
\hline$x_{8}$ & $m_{1}, m_{5}, m_{8}, m_{9}$ & $t_{2}, t_{3}, t_{6}, t_{34}, t_{41}, t_{42}, t_{48}, t_{50}$ & \\
\hline$x_{9}$ & $m_{1}, m_{5}, m_{8}, m_{9}$ & $t_{3}, t_{6}, t_{20}, t_{34}, t_{41}, t_{42}, t_{48}, t_{50}$ & \\
\hline$x_{10}$ & $m_{1}, m_{8}, m_{9}$ & $t_{2}, t_{3}, t_{6}, t_{15}, t_{34}, t_{42}, t_{50}$ & \\
\hline$x_{11}$ & $m_{1}, m_{8}, m_{9}$ & $t_{3}, t_{6}, t_{15}, t_{20}, t_{34}, t_{42}, t_{50}$ & \\
\hline$x_{12}$ & $m_{1}, m_{6}, m_{7}, m_{9}$ & $t_{34}, t_{42}$ & \\
\hline$x_{13}$ & $m_{1}, m_{6}, m_{9}$ & $t_{2}, t_{3}, t_{6}, t_{15}, t_{34}, t_{42}$ & \\
\hline$x_{14}$ & $m_{1}, m_{6}, m_{9}$ & $t_{3}, t_{6}, t_{15}, t_{20}, t_{34}, t_{42}$ & \\
\hline$x_{15}$ & $m_{1}, m_{5}, m_{6}, m_{9}$ & $t_{2}, t_{3}, t_{6}, t_{34}, t_{36}, t_{42}, t_{49}$ & \\
\hline$x_{16}$ & $m_{1}, m_{5}, m_{6}, m_{9}$ & $t_{3}, t_{6}, t_{20}, t_{34}, t_{36}, t_{42}, t_{49}$ & \\
\hline$x_{17}$ & $m_{1}, m_{5}, m_{6}, m_{9}$ & $t_{2}, t_{3}, t_{6}, t_{34}, t_{36}, t_{41}, t_{42}$ & \\
\hline$x_{18}$ & $m_{1}, m_{5}, m_{6}, m_{9}$ & $t_{3}, t_{6}, t_{20}, t_{34}, t_{36}, t_{41}, t_{42}$ & \\
\hline$x_{19}$ & $m_{1}, m_{5}, m_{6}, m_{9}$ & $t_{2}, t_{3}, t_{6}, t_{34}, t_{41}, t_{42}, t_{48}, t_{50}$ & \\
\hline$x_{20}$ & $m_{1}, m_{5}, m_{6}, m_{9}$ & $t_{3}, t_{6}, t_{20}, t_{34}, t_{41}, t_{42}, t_{48}, t_{50}$ & \\
\hline$x_{21}$ & $m_{1}, m_{3}, m_{6}, m_{9}$ & $t_{2}, t_{3}, t_{6}, t_{34}, t_{42}$ & \\
\hline$x_{22}$ & $m_{1}, m_{3}, m_{6}, m_{9}$ & $t_{3}, t_{6}, t_{20}, t_{34}, t_{42}$ & \\
\hline$x_{23}$ & $m_{1}, m_{7}, m_{8}, m_{9}$ & $t_{34}, t_{42}, t_{50}$ & \\
\hline$x_{24}$ & $m_{1}, m_{2}, m_{5}, m_{7}, m_{9}, m_{10}$ & $t_{2}, t_{34}, t_{42}$ & \\
\hline$x_{25}$ & $m_{1}, m_{2}, m_{5}, m_{7}, m_{9}, m_{10}$ & $t_{20}, t_{34}, t_{42}$ & \\
\hline$x_{26}$ & $m_{1}, m_{3}, m_{8}, m_{9}$ & $t_{2}, t_{3}, t_{6}, t_{34}, t_{42}, t_{50}$ & \\
\hline$x_{27}$ & $m_{1}, m_{3}, m_{8}, m_{9}$ & $t_{3}, t_{6}, t_{20}, t_{34}, t_{42}, t_{50}$ & \\
\hline$x_{28}$ & $m_{1}, m_{5}, m_{9}, m_{10}$ & $t_{2}, t_{3}, t_{6}, t_{34}, t_{36}, t_{41}$ & \multirow{8}{*}{$c_{2}$} \\
\hline$x_{29}$ & $m_{1}, m_{5}, m_{7}, m_{9}, m_{10}$ & $t_{2}, t_{34}, t_{36}, t_{41}$ & \\
\hline$x_{30}$ & $m_{1}, m_{5}, m_{9}, m_{10}$ & $t_{3}, t_{6}, t_{20}, t_{34}, t_{36}, t_{41}$ & \\
\hline$x_{31}$ & $m_{1}, m_{5}, m_{7}, m_{9}, m_{10}$ & $t_{20}, t_{34}, t_{36}, t_{41}$ & \\
\hline$x_{32}$ & $m_{1}, m_{5}, m_{9}, m_{10}$ & $t_{2}, t_{3}, t_{6}, t_{41}, t_{48}, t_{50}$ & \\
\hline$x_{33}$ & $m_{1}, m_{5}, m_{9}, m_{10}$ & $t_{3}, t_{6}, t_{20}, t_{41}, t_{48}, t_{50}$ & \\
\hline$x_{34}$ & $m_{1}, m_{5}, m_{7}, m_{9}, m_{10}$ & $t_{2}, t_{41}, t_{48}, t_{50}$ & \\
\hline$x_{35}$ & $m_{1}, m_{5}, m_{7}, m_{9}, m_{10}$ & $t_{20}, t_{41}, t_{48}, t_{50}$ & \\
\hline$x_{36}$ & $m_{10}$ & $t_{2}, t_{15}$ & \multirow{2}{*}{$c_{3}$} \\
\hline$x_{37}$ & $m_{10}$ & $t_{15}, t_{20}$ & \\
\hline$x_{38}$ & $m_{5}, m_{9}, m_{10}$ & $t_{2}, t_{34}, t_{36}, t_{49}$ & \multirow{2}{*}{$c_{4}$} \\
\hline$x_{39}$ & $m_{5}, m_{9}, m_{10}$ & $t_{20}, t_{34}, t_{36}, t_{49}$ & \\
\hline$x_{40}$ & $m_{3}, m_{10}$ & $t_{2}$ & \multirow{2}{*}{$c_{5}$} \\
\hline$x_{41}$ & $m_{3}, m_{10}$ & $t_{20}$ & \\
\hline$x_{42}$ & - & $t_{48}, t_{49}, t_{50}$ & $c_{6}$ \\
\hline
\end{tabular}


Table 7: t-clusters

\begin{tabular}{|c|c|}
\hline ID & Description \\
\hline$c_{1}$ & $\begin{array}{l}\text { processes related to immune system response with iNOS obtained by } \\
\text { cytokines and peroxynitrite } \mathrm{OONO}^{-} \text {anion (do not influence atheroscle- } \\
\text { rosis progression) }\end{array}$ \\
\hline$c_{2}$ & $\begin{array}{l}\text { atherosclerosis progression influenced by processes related to immune } \\
\text { system response with iNOS obtained by mast cells degranulation }\end{array}$ \\
\hline$c_{3}$ & atherosclerosis progression influenced by rich cholesterol diet \\
\hline$c_{4}$ & $\begin{array}{l}\text { atherosclerosis progression influenced by expression of ICAM1 based on } \\
\text { lipids peroxidation by peroxynitrite }\end{array}$ \\
\hline$c_{5}$ & atherosclerosis progression influenced by inflammatory process \\
\hline$c_{6}$ & endothelium activation influenced by NO \\
\hline
\end{tabular}

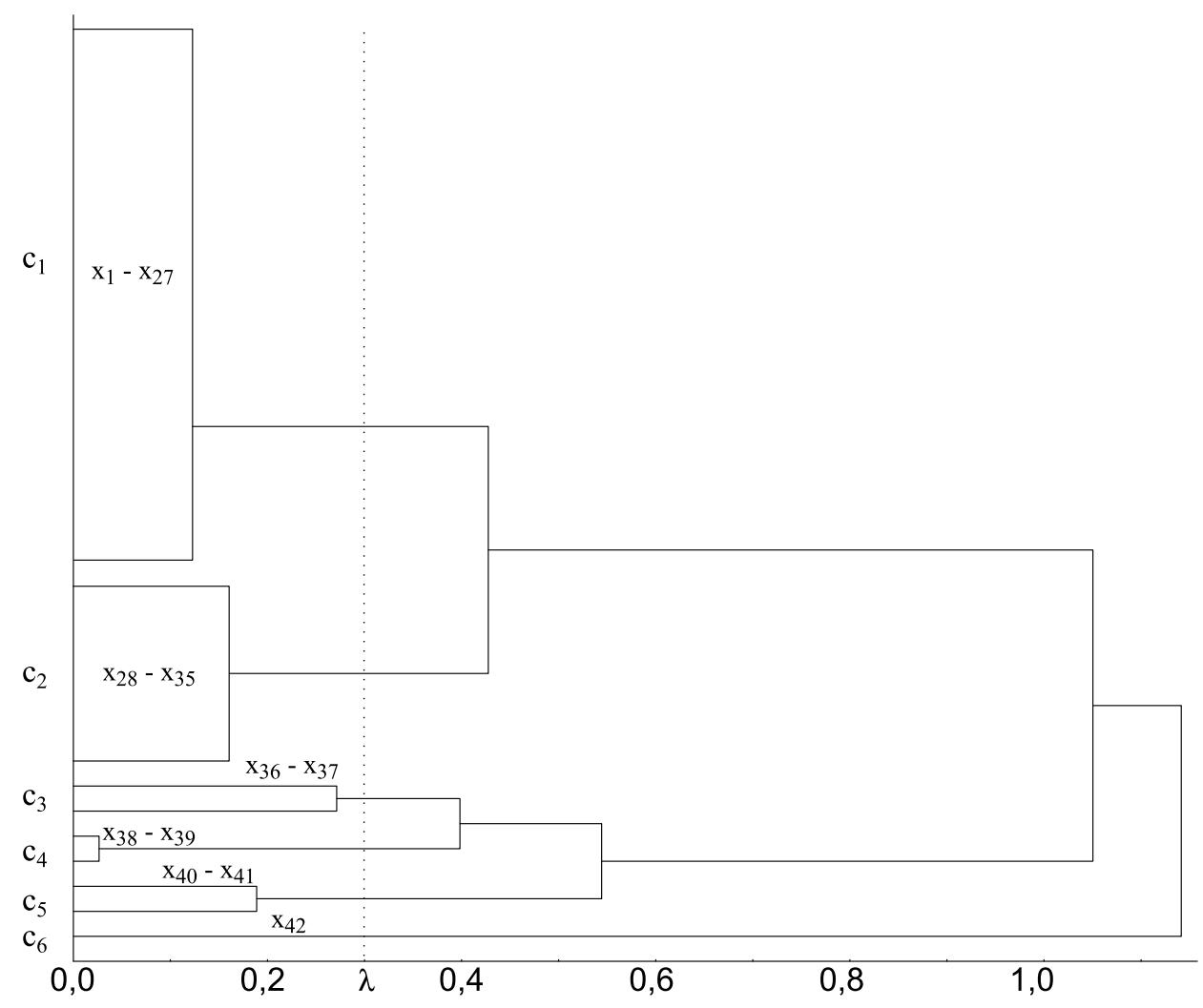

Figure 2: t-clusters dendrogram. t-invariants were clustered as 51-dimensional vectors (51 transitions) using UPGMA linkage with 1-Pearson $\mathrm{R}$ correlation metric. 